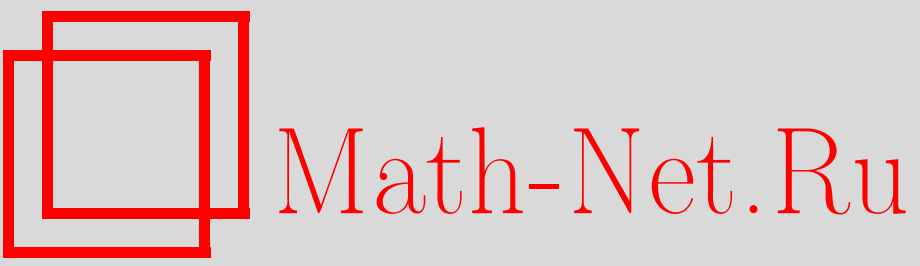

А. А. Чубатов, В. Н. Кармазин, Экспресс-контроль за источником загрязнения атмосферы на основе метода последовательной функциональной аппроксимации, Вестн. Сам. гос. техн. ун-та. Сер. Физ.-мат. науки, 2008, выпуск 2(), 210-214

DOI: https://doi.org/10.14498/vsgtu599

Использование Общероссийского математического портала Math-Net.Ru подразумевает, что вы прочитали и согласны с пользовательским соглашением http://www. mathnet.ru/rus/agreement

Параметры загрузки:

IP : 34.227 .88 .159

26 апреля 2023 г., 17:11:05 
УДК 517.956.47:519.642.5

\section{ЭКСПРЕСС-КОНТРОЛЬ ЗА ИСТОЧНИКОМ ЗАГРЯЗНЕНИЯ АТМОСФЕРЫ НА ОСНОВЕ МЕТОДА ПОСЛЕДОВАТЕЛЬНОЙ ФУНКЦИОНАЛЬНОЙ АППРОКСИМАЦИИ}

\section{А. А. Чубатов, В. Н. Кармазин}

Кубанский государственный университет, 350040, г. Краснодар, ул. Ставропольская, 149.

E-mails: chaa@inbox.ru, karmazin@kubsu.ru

Предлагается подход, позволяющий последовательно оценивать интенсивность действия источника загрязнения атмосферы на основе данных измерений кончентращии примеси в нескольких стационарных пунктах контроля. Обратная задача решалась методом последовательной бункииональной аппроксимации. Решение представлено в виде иифрового фильтра.

Ключевые слова: обратная задача, некорректная задача, устойчивость, регуляризачия, последовательная функииональная аппроксимация, загрязнение атмосферы, уравнение турбулентной дифбузии.

Введение. Для описания процессов распространения примеси в атмосфере используем двумерное линейное уравнение турбулентной диффузии [1] с однородными начальным и граничными условиями:

$$
\frac{\partial q}{\partial t}+\frac{\partial}{\partial x}\left(v_{x} q\right)+\frac{\partial}{\partial y}\left(v_{y} q\right)=\frac{\partial}{\partial x}\left(K_{x} \frac{\partial q}{\partial x}\right)+\frac{\partial}{\partial y}\left(K_{y} \frac{\partial q}{\partial y}\right)+f(x, y) \cdot g(t)
$$

где $q=q(x, y, t)$ - интегральная по высоте концентрация примеси, $\left(v_{x} ; v_{y}\right)$ вектор скорости ветра, $\left(K_{x} ; K_{y}\right)$ - вектор коэффициентов турбулентной диффузии, $f(x, y)$ - функция, характеризующая пространственное расположение источника загрязнения, $g(t)$ - интенсивность действия источника.

Обратная задача идентификации интенсивности выбросов источника состоит в последовательном определении функции $g(t)$ по данным измерений концентрации в стационарных пунктах контроля, расположенных в точках $\left(x_{j}, y_{j}\right), j=1,2, \ldots, J$. Измерения проводятся через промежутки времени $\Delta t$.

Будем считать, что ошибка замеров концентрации аддитивна:

$$
c_{j i}=q\left(x_{j}, y_{j}, t_{i}\right)+\delta \cdot \gamma,
$$

где $c_{j i}$ - концентрация, измеренная $j$-тым датчиком в момент времени $t_{i}=$ $=i \cdot \Delta t, \delta$ - среднеквадратичная ошибка измерений датчиков, $\gamma-$ нормальная случайная величина $(M(\gamma)=0, D(\gamma)=1)$.

Обратная задача для источника характеризуется неустойчивостью решения к погрешностям замеров концентрации и требует специальных методов решения [2-4]. Для решения задачи использовались методы шаговой регуляризации и последовательной функциональной аппроксимации $[3,4]$.

1. Метод последовательной функциональной аппроксимации. Линейность задачи (1) позволяет воспользоваться численным аналогом теоремы Дюаме-

Чубатов Андрей Алексеевич - аспирант кафедры прикладной математики Кубанского государственного университета.

Кармазин Владимир Николаевич - профессор кафедры прикладной математики Кубанского государственного университета; к.ф.-м.н., доцент. 
ля:

$$
q\left(x_{j}, y_{j}, t_{i}\right)=\sum_{n=1}^{i} g\left(t_{n}\right) \cdot\left(\mathcal{Q}\left(x_{j}, y_{j}, t_{i-n+1}\right)-\mathcal{Q}\left(x_{j}, y_{j}, t_{i-n}\right)\right)
$$

где $\mathcal{Q}(x, y, t)$ - решение прямой задачи $(1)$ при $g(t)=1$ и однородных начальном и граничных условиях.

Введём обозначения:

$$
q\left(x_{j}, y_{j}, t_{i}\right)=q_{j i}, \quad \mathcal{Q}\left(x_{j}, y_{j}, t_{i}\right)=\phi_{j i}, \quad \phi_{j(i+1)}-\phi_{j i}=\Delta \phi_{j i} .
$$

Величина $\phi_{j i}$ называется ступенчатым коэффициентом чувствительности, а величина $\Delta \phi_{j i}$ - импульсным коэффициентом чувствительности.

Пусть $g(t)$ принимает на каждом промежутке $[(N-1) \cdot \Delta t ; N \cdot \Delta t]$ постоянное значение $g_{N}$. Считая $g_{1}, g_{2}, \ldots, g_{N-1}$ известными величинами, вычисленными на предыдущих шагах, оценим $g_{N}$. Для придания устойчивости решению обратной задачи рассмотрим $g(t)$ на нескольких $(r)$ временных промежутках сразу. Будем считать, что $g_{N}, g_{N+1}, \ldots, g_{N+r-1}$ связаны некоторой функциональной зависимостью. При $r=1$ получается метод шаговой регуляризации.

Используя (2) для моментов времени $t_{N}, t_{N+1}, \ldots, t_{N+r-1}$, запишем матричное уравнение

$$
\mathbf{Q}=\left.\mathbf{Q}\right|_{\mathbf{g}=\mathbf{0}}+\mathbf{\Phi} \cdot \mathbf{G}
$$

где $\mathbf{Q},\left.\mathbf{Q}\right|_{\mathbf{g}=\mathbf{0}} \in \mathbb{R}^{r \cdot J}, \mathbf{\Phi} \in \mathbb{R}^{(r \cdot J) \times r}, \mathbf{G} \in \mathbb{R}^{r}$,

$$
\begin{aligned}
& \mathbf{Q}=\left(\begin{array}{l}
\mathbf{Q}(N) \\
\mathbf{Q}(N+1) \\
\cdots \\
\mathbf{Q}(N+r-1)
\end{array}\right), \quad \mathbf{Q}(i)=\left(\begin{array}{c}
q_{1 i} \\
q_{2 i} \\
\cdots \\
q_{J i}
\end{array}\right), \quad \mathbf{G}=\left(\begin{array}{c}
g_{N} \\
g_{N+1} \\
\cdots \\
g_{N+r-1}
\end{array}\right), \\
& \left.\mathbf{Q}\right|_{\mathbf{g}=\mathbf{0}}=\left(\begin{array}{l}
\left.\mathbf{Q}\right|_{\mathbf{g}=\mathbf{0}}(0) \\
\left.\mathbf{Q}\right|_{\mathbf{g}=\mathbf{0}}(1) \\
\cdots \\
\left.\mathbf{Q}\right|_{\mathbf{g}=\mathbf{0}}(r-1)
\end{array}\right),\left.\quad \mathbf{Q}\right|_{\mathbf{g}=\mathbf{0}}(k)=\left(\begin{array}{c}
\sum_{n=1}^{N-1} g_{n} \cdot \Delta \phi_{1(N+k-n)} \\
\ldots \\
\sum_{n=1}^{N-1} g_{n} \cdot \Delta \phi_{J(N+k-n)}
\end{array}\right), \\
& \boldsymbol{\Phi}=\left(\begin{array}{cccc}
\phi(0) & & & \\
\phi(1) & \phi(0) & & \\
\vdots & \vdots & \ddots & \\
\phi(r-1) & \phi(r-2) & \cdots & \phi(0)
\end{array}\right), \quad \phi(k)=\left(\begin{array}{c}
\Delta \phi_{1 k} \\
\Delta \phi_{2 k} \\
\cdots \\
\Delta \phi_{J k}
\end{array}\right)
\end{aligned}
$$

Минимизируем сумму квадратов разностей между измеренными $\mathbf{C}$ и расчётными $\mathbf{Q}$ значениями концентрации:

$$
S=(\mathbf{C}-\mathbf{Q})^{\mathrm{T}} \cdot(\mathbf{C}-\mathbf{Q}) \rightarrow \min _{G}
$$

где $\mathbf{C} \in \mathbb{R}^{r \cdot J}$,

$$
\mathbf{C}=\left(\begin{array}{l}
\mathbf{C}(N) \\
\mathbf{C}(N+1) \\
\cdots \\
\mathbf{C}(N+r-1)
\end{array}\right), \quad \mathbf{C}(i)=\left(\begin{array}{c}
c_{1 i} \\
c_{2 i} \\
\cdots \\
c_{J i}
\end{array}\right)
$$

Временно предположим, что $g_{N+1}, g_{N+2}, \ldots, g_{N+r-1}$ выражаются через некоторую функциональную зависимость от $g_{N}$ и $g_{N-1}, g_{N-2}, \ldots, g_{N-p}$, оце- 
ним единственную неизвестную $g_{N}$ и перейдем к следующему шагу, предполагая зависимость $g_{(N+1)+1}, g_{(N+1)+2}, \ldots, g_{(N+1)+r-1}$ от $g_{(N+1)}$ и

$g_{(N+1)-1}, g_{(N+1)-2}, \ldots, g_{(N+1)-p}$.

Пусть эта функциональная зависимость имеет вид

$$
\mathbf{G}=\mathbf{A} \cdot g_{N}+\mathbf{B} \cdot \mathbf{G}_{\mathbf{0}}, \quad \mathbf{G}_{\mathbf{0}}=\left(\begin{array}{l}
g_{N-1} \\
g_{N-2} \\
\cdots \\
g_{N-p}
\end{array}\right),
$$

где $\mathbf{A} \in \mathbb{R}^{r}, \mathbf{B} \in \mathbb{R}^{r \times p}$, для обеспечения условия $G_{1}=g_{M}$ при любом виде функциональной зависимости $A_{1}=1, B_{1 n}=0$.

Нами рассмотрен простейший случай - в предположении постоянства $g(t)$ в течение $r$ последовательных промежутков времени

$$
g_{N}=g_{N+1}=\cdots=g_{N+r-1},
$$

а также случай линейной зависимости между $g_{N-1}, g_{N}, g_{N+1}, \ldots, g_{N+r-1}$ :

$$
g_{N+i-1}=g_{N}+(i-1) \cdot\left(g_{N}-g_{N-1}\right)=i \cdot g_{N}+(1-i) \cdot g_{N-1} .
$$

С учётом (5) и (3) решение задачи (4) имеет вид

$$
g_{N}=\left((\boldsymbol{\Phi} \cdot \mathbf{A})^{\mathrm{T}} \cdot(\boldsymbol{\Phi} \cdot \mathbf{A})\right)^{-1} \cdot(\mathbf{\Phi} \cdot \mathbf{A})^{\mathrm{T}} \cdot\left(\mathbf{C}-\left.\mathbf{Q}\right|_{\mathbf{g}=\mathbf{0}}-\mathbf{\Phi} \cdot \mathbf{B} \cdot \mathbf{G}_{\mathbf{0}}\right) .
$$

Решение (8) является линейной функцией измеренных концентраций $c_{j i}$ $i=1,2, \ldots, N+r-1$, и его можно представить в виде цифрового фильтра $[4,5]$

$$
g_{N}=\sum_{i=1}^{N+r-1} \sum_{j=1}^{J} f_{j(N-i)} \cdot c_{j i},
$$

где $f_{j(i-r)}$ - коэффициенты фильтра, $f_{j(i-r)}=\mathcal{G}_{j i}, i=1,2, \ldots, N+r-1$, $\mathcal{G}_{j i}$ - решение (8) обратной задачи при $c_{j r}=1 ; c_{j i}=0, i \neq r$.

Решение в форме цифрового фильтра в вычислительном отношении эффективнее других форм, т. к. коэффициенты $f_{j k}$ вычисляются один раз.

2. Результаты вычислительных экспериментов. На ряде методических задач проведено множество численных экспериментов. Построены устойчивые численные приближения к искомым интенсивностям для источников различных типов (точечных, линейных, площадных), в том числе и при наличии ошибок измерений $\left(\delta=0 \div 0,03 \cdot q_{\max }\right)$. Для учета качества восстановления интенсивности $g(t)$ использовалась среднеквадратическая погрешность

$$
\sigma_{G}=\sqrt{\frac{1}{N_{\max }} \sum_{n=1}^{N_{\max }}\left(g\left(\left(n-\frac{1}{2}\right) \cdot \Delta t\right)-g_{n}\right)^{2}} .
$$

Для каждого датчика существует критический шаг $\Delta t_{\mathrm{st}[1]}$ такой, что при шаге решения обратной задачи $\Delta t>\Delta t_{\mathrm{st}[1]}$ решение устойчиво, т.е. имеет место эффект шаговой регуляризации. Но возможности ее ограничены, т.к. для некоторых датчиков $\Delta t_{\mathrm{st}[1]}$ может быть довольно большим, в результате 
чего восстановленное решение загрубляется. При $r=1$ с возрастанием $\Delta t$ наблюдается ослабление зависимости величины $\sigma_{G}$ от параметра $\delta$ (см. рис. 1$)$. При использовании нескольких датчиков $(J>1)$ датчик с меньшим $\Delta t_{\mathrm{st}[1]}$ имеет преобладающее влияние. Использование двух датчиков с одинаковыми $\Delta t_{\mathrm{st}[1]}$ улучшает результат по сравнению с использованием одного датчика.

Желание повысить точность восстановления интенсивности, уменьшая шаг по времени, приводит к неустойчивости решения обратной задачи. Использование функциональной аппроксимации с несколькими последовательными шагами по времени $(r>1)$ позволяет получать устойчивые оценки интенсивности при $\Delta t<\Delta t_{\mathrm{st}[1]}$. Например, при $r=3$ приемлемые результаты получаются при шаге по времени $\Delta t_{\mathrm{st}[3]}=\Delta t_{\mathrm{st}[1]} / 4$ (см. табл.).

Примерные значения $\Delta t_{\mathrm{st}[r]}$ для некоторых $r$

\begin{tabular}{c|c|c|c|c|c}
\hline$r$ & 1 & 2 & 3 & 4 & 6 \\
\hline$\Delta t_{\mathrm{st}[r]}$ & $\Delta t_{\mathrm{st}[1]}$ & $\Delta t_{\mathrm{st}[1]} / 3$ & $\Delta t_{\mathrm{st}[1]} / 4$ & $\Delta t_{\mathrm{st}[1]} / 6$ & $\Delta t_{\mathrm{st}[1]} / 10$ \\
\hline
\end{tabular}

В рамках проведённых экспериментов не обнаружено существенных различий при использовании функциональных зависимостей (6) и (7).

При $\Delta t=$ const с возрастанием $r$ влияние параметра $\delta$ на величину $\sigma_{G}$ ослабевает и при определённом $r=r_{c}$ величина $\sigma_{G}$ практически не зависит от $\delta \in\left[0 ; 0,03 \cdot q_{\max }\right]$ (см. рис. 2$)$.

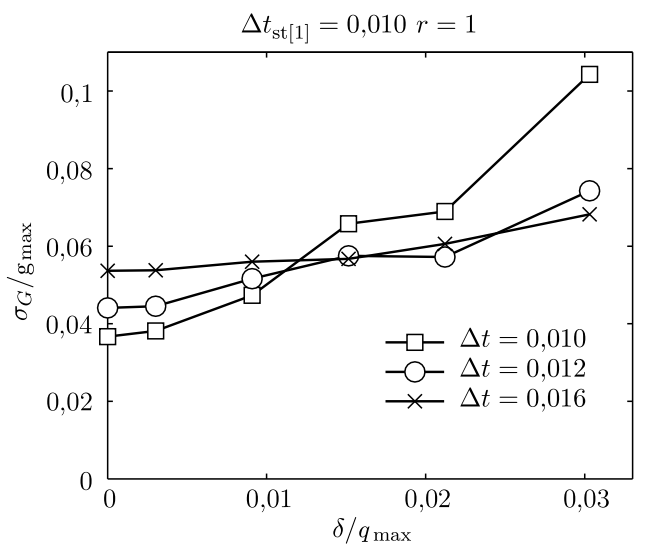

Рис. 1. Зависимость $\sigma_{G}$ от $\Delta t$ и $\delta$ при

$$
r=1
$$

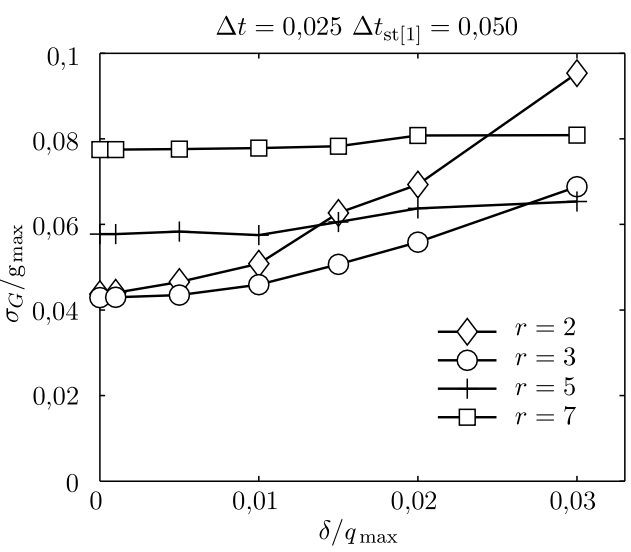

Рис. 2. Зависимость $\sigma_{G}$ от $r$ и $\delta$ при $\Delta t=$ const $=\Delta \mathrm{t}_{\mathrm{st}[1]} / 2$

Анализ результатов численных экспериментов позволяет сделать вывод, что для пары чисел $\left(\Delta t / \Delta t_{\mathrm{st}[1]}, \delta\right), \Delta t / \Delta t_{\mathrm{st}[1]} \in[0,1 ; 1], \delta \in\left[0 ; 0,03 \cdot q_{\max }\right]$ можно подобрать $r$, при котором погрешность восстановления интенсивности минимальна. В этой связи актуальна разработка алгоритмических методов выбора параметра $r$, обеспечивающего получение устойчивых оценок интенсивности.

В рассмотренном методе информация о замерах концентрации с датчиков усваивается последовательно, что позволяет организовать on-line контроль за выбросами загрязнений из источника в атмосферу.

Работа выполнена при поддержке Российского фонда фундаментальных исследований и администрации Краснодарского края (проект № 06-01-96643). 


\section{БИБЛИОГРАФИЧЕСКИЙ СПИСОК}

1. Марчук, Г. И. Математическое моделирование в проблеме окружающей среды [Текст] Г. И. Марчук. - М.: Наука, 1982. - 320 с.

2. Тихонов, A. Н. Методы решения некорректных задач [Текст] / А. Н. Тихонов, В. Я. Арсенин. - М.: Наука, 1986. - 286 с.

3. Алифанов, О. М. Обратные задачи теплообмена [Текст] / О. М. Алифанов. - М.: Машиностроение, 1988. - 280 с. - ISBN 5-217-00134-8.

4. Бек, Дж. Некорректные обратные задачи теплопроводности: Пер. с англ. [Текст] / Дж. Бек, Б. Блакуэлл, С. Чент-Клэр, мл. - М.: Мир, 1989. - 312 с. - ISBN 5-030009140.

5. Хемминг, Р. В. Цифровые фильтры: Пер. с англ. [Текст] / Р. В. Хемминг. - М.: Советское радио, 1980. - 224 с.

Поступила в редакцию $15 / \mathrm{VII} / 2008$;

в окончательном варианте $-27 / \mathrm{X} / 2008$.

MSC: 90C39, 90C15

\section{EXPRESS-CONTROL OF ATMOSPHERIC POLLUTION SOURCE ON THE BASIS OF SERIAL FUNCTIONAL SPECIFICATION}

\section{A. A. Chubatov, V. N. Karmazin}

Kuban State University, //350040, Krasnodar, Stavropolskaya st., 149.

E-mails: chaa@inbox.ru, karmazin@kubsu.ru

The approach described allows to estimate the intensity of atmospheric pollution source step-by step on the basis of impurities concentration measurements in several stationary control points. The inverse problem was solved with the help of sequential functional specification method. The solution is presented in the form of a digital filter.

Key words: inverse problem, ill-posed problem, stability, regularization, function specification method, sequential functional approximation, atmospheric pollution, turbulent diffusion equation.

Original article submitted 15/VII/2008; revision submitted $27 / \mathrm{X} / 2008$.

Chubatov Andrey Alexeevich, Post graduate student, Dept. of applied mathematics of Kuban State University.

Karmazin Vladimir Nikolaevich, Ph. D. (Phis. ES Math.) Prof., Dept. of applied mathematics of Kuban State University. 